\title{
Interworking of Self-organizing Hierarchical Ad Hoc Networks and the Internet*
}

\author{
Hyukjoon Lee ${ }^{1}$, Seung Hyong Rhee ${ }^{1}$, Dipankar Raychaudhuri ${ }^{2}$, and Wade Trappe ${ }^{2}$ \\ ${ }^{1}$ Kwangwoon University, 447-1 Wolgye-Dong, Nowon-Gu, Seoul 139-701, Korea \\ \{hlee, shrhee\} @daisy.kw.ac.kr \\ ${ }^{2}$ WINLAB, Rutgers University, 73 Brett Road, Piscataway, NJ 08854, USA \\ \{ray, trappe\}@winlab.rutgers.edu
}

\begin{abstract}
Self-organizing hierarchical ad hoc network (SOHAN) is a new network architecture that has been proposed to increase the scalability property of flat ad hoc networks. This paper describes how SOHAN interoperates efficiently with the Internet based on IPv6. Procedures for the autoconfiguration of a globally-routable address, routing and gateway discovery are presented. The amount of control overheads is reduced by taking advantage of cross-layer interaction and limited-scope broadcast (LSBC) techniques. Simulation results display SOHAN with the proposed interworking procedures outperforms conventional flat ad hoc networks in interworking with the Internet in terms of throughput capacity and scalability.
\end{abstract}

Keywords: Ad hoc network, IPv6, Mobile IP, Interworking, Cross-layer interaction.

\section{Introduction}

Ad hoc networks have been studied extensively by the research community during the past several years motivated by their high potential for rapid deployment and cost benefits. The focus of most research works has been on stand-alone "flat" networks, in which no hierarchical relationship between nodes is assumed and every node contributes to multi-hop communication. One of the fundamental problems of the flat wireless networks is that they do not scale well. Gupta and Kumar describe that the throughput of a wireless network is bounded above and decreases as $o(1 / \sqrt{n})$ as $n$ becomes large [1]. This motivates the investigation of a new wireless network architecture based on a hierarchical structure.

SOHAN (Self-Organizing Hierarchical Ad hoc Network) is a novel ad hoc network architecture proposed by Ganu et al. that consists of three tiers, i.e. access points (AP's), forwarding nodes (FN's) and mobile nodes (MN's) [2]. In this new architecture, the AP's are connected together by high-speed wired links. Using highspeed wired links not only increases the system capacity, but also provides a convenient framework for interconnecting to a wired network. This paper describes how SOHAN interoperates efficiently with the Internet based on IPv6.

* This work was supported by Grant No. R01-2001-00349 from the Korea Science \& Engineering Foundation and Research Grant of Kwangwoon University in 2004. 
The interworking of ad hoc networks with the Internet presents interesting challenges. For example, the global routing of the Internet cannot be directly applied, since each type of networks uses a different address architecture (i.e., flat vs. hierarchical) and routing protocols (i.e., host-specific vs. prefix matching). There exist several research works published in the literature that propose different approaches to interconnect the ad hoc networks and the Internet [3-8]. Most of them use Mobile IP to provide the mobile nodes (MN's) with globally-routable addresses, i.e., care-of addresses (CoA's) [3-9]. In this approach, a foreign agent (FA) acts as the interworking gateway. One of the main concerns of this approach is that it requires flooding-based operations. For example, duplicate address detection (DAD) in address autoconfiguration, gateway discovery and route discovery flood messages over the entire ad hoc networks. Flooding could decrease the throughput capacity of the network significantly.

The interworking methods for SOHAN proposed in this work takes advantage of the hierarchical structure and routing mechanism such that efficient interworking is achieved without interworking gateways. Based on the expectation that IPv6 would become the unifying packet transport protocol in both the core and access networks of the future communication systems, we focus on the interworking with IPv6.

The rest of this paper is organized as follows. In section 2, we introduce SOHAN architecture. In section 3, we present the detailed discussion on how the interworking can be achieved. In section 4 , we show some simulation results. Finally, in section 5, we conclude our discussion.

\section{Self-organizing Hierarchical Ad Hoc Networks}

\subsection{Architecture}

SOHAN is a novel self-organizing hierarchical ad hoc network architecture designed to provide significant improvements in systems capacity and performance relative to conventional float ad hoc networks. It consists of three tiers of radio nodes: lowpower mobile node $(\mathrm{MN})$ at the lowest tier, high-power forwarding nodes $(\mathrm{FN})$ at the mid-tier, and wired access points (AP) at the highest tier (Fig. 1).

The MN operates on a single radio (e.g. 802.15.4) and, instead of directly connecting to other MN's, it connects to an AP or FN of the best link quality. As a user device, the MN does not forward packets for other nodes. The bandwidth and energy-constrained MN's do not communicate with an Internet host, but merely send/receive their data to/from the directly-connected AP's or FN's of much higher bandwidth and energy.

The FN can have a direct radio connection with all three types of nodes. The main function of $\mathrm{FN}$ is to forward packets for other nodes by multi-hop ad hoc routing. It can be equipped with a single or two radio interfaces, one for FN-MN traffic and the other for the intra-FN and FN-AP traffic. The FN can be either fixed or mobile. The AP has both a radio interface (e.g. 802.11) and a wired interface to the wired links. The AP's can be configured as an access router (AR). Multiple FN's can be directly connected to the AP in ad hoc mode. The transmission speed of the wired links that connect the AP's is assumed orders of magnitude faster than that of the wireless links. 


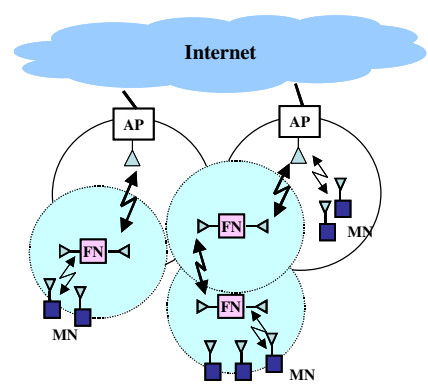

Fig. 1. Self-Organizing Hierarchical Ad hoc Network (SOHAN) architecture

\begin{tabular}{|c|c|c|c|c|c|c|c|}
\hline $\begin{array}{c}\text { Msg } \\
\text { Type }\end{array}$ & $\begin{array}{c}\text { Node } \\
\text { Type }\end{array}$ & $\begin{array}{c}\text { Node } \\
\text { ID }\end{array}$ & Channel & $\begin{array}{c}\text { Cost to } \\
\text { AP }\end{array}$ & $\begin{array}{c}\text { Beacon } \\
\text { No. }\end{array}$ & BSSID & $\begin{array}{c}\text { AP IPv6 } \\
\text { Addr. }\end{array}$ \\
\hline
\end{tabular}

Fig. 2. Message format of SOHAN beacon and association

\subsection{Topology Discovery}

The AP's and FN's use a self-organizing topology discovery protocol based on beacons. The beacon is an application-level message based on the 802.11 MAC beacons and used by the AP's and FN's to identify their one-hop neighbors. It carries information about the quality of physical links to other nodes within a radio range, which is used as the basis of determining the optimal logical network topology.

Upon bootstrapping, all nodes enter a self-organizing phase by transmitting the beacons on their predetermined channels and repeat this phase periodically. Based on the beacons received, the FN's and MN's update their neighbor table and transmit the association messages to the best one-hop parent. The beacon and association messages share the same augmented message format (Fig. 2).

\subsection{Routing}

The routing protocol proposed in [2] is based on the topology discovery protocol. The neighbor table generated by the topology discovery protocol contains information about the next hop node (i.e., parent node) for each node to reach an AP. The neighbor tables are periodically exchanged between neighboring devices such that information about multi-hop paths can be incrementally built up in a similar way to the distance-vector routing protocol. The main purpose of this protocol is to enable data to flow from the MN's towards the AP's. Hence, entries for the MN's are excluded from the neighbor table exchange. A packet sent across the boundaries of two subtrees would be routed via the wired links. This routing strategy is based on the assumption that the wired links connecting the AP's provide much higher bandwidth than the wireless links. It may be sub-optimal since an ad-hoc path may exist that goes through the FN's in less number of hops.

More recently, a new L2.5 routing protocol based on AODV with appropriate modifications has been proposed [10]. This routing protocol, operating with the MAC addresses, sets up the initial routing table based on the neighbor table and adds an entry as a new route is found on-demand. The routes from the AP to the FN/MN's are found by flooding RREQ (Route Request) messages or by reverse route setup when data packets are transmitted by the FN's towards the AP. 


\section{Interworking of SOHAN and the Internet}

The topology discovery and routing protocols discussed in the previous section can make the SOHAN appear as a set of Ethernet-like wireless LAN segments. Therefore, IPv6 operations used in interworking, such as router discovery and address autoconfiguration, can be directly applied in SOHAN (Fig. 3). In what follows, we assume that every AP is configured as an IPv6 router in order to simply our discussion. All aspect of the interworking functionalities discussed in this paper can easily be extended with minor modifications when multiple AP's exist in a subnet.

\subsection{Address Autoconfiguration}

An FN/MN in SOHAN must be configured with a globally-routable IP address to communicate with an Internet host. IPv6 stateless address autoconfiguration allows a globally-routable IP address for the FN/MN to be constructed from the prefix of an AR when the FN/MN joins or boots up in an ad hoc network [11]. If the FN/MN does not have its own IPv6 home address, the FN/MN should temporarily configure an initial address simply by forming a link-local address, or using the IPv6_MANET initial prefix [8]. The uniqueness of these tentative addresses can be verified using the strong DAD described in $[12,13]$.

DAD operation based on NDP, when applied to an ad hoc network, must be performed in a multi-hop fashion over the entire network (i.e., flooding), if a single interworking gateway is used. However, flooding causes scalability problem when there are a large number of nodes in the network. Moreover, it is meaningless to flood the DAD packets to the nodes with possibly different prefixes (i.e., the nodes associated with different AR's) as in case of SOHAN. Therefore, the flooding in SOHAN is confined within the subset of nodes that are associated with the same AP. This so-called limited-scope broadcast (LSBC) uses the information about each node's association with AP's stored in the neighbor table of the MAC layer. The node can determine whether it is associated with the same AP as the source of flooding by checking the Basic Service Set ID (BSSID).

The globally routable IP address is formed by appending to the prefix of the AP the interface identifier of the FN/MN. We modify the format of a beacon message specified in [2] to include the IPv6 address of the AP associated with each FN (Fig. 2). The FN/MN that is multi-hop away from an AP receives the address of the AP associated with its upstream neighbor. Thus, the periodic broadcast of router advertisement is not used. Therefore, a considerable amount of valuable radio resource can be saved.

\subsection{Routing}

Address autoconfiguration discussed above logically maps a part of the network corresponding to a subtree with an AP as its root in the network topology to an IP subnet (Fig. 4). Since the ad hoc routing is performed below the network layer, the subtree appears as a single-hop wireless LAN segment to the IPv6. The interworking between the two routing protocols becomes a cross-layer interaction problem. 
Since the FN/MN's use MAC addresses as their identifier for ad hoc routing at L2.5, the IP addresses must be translated to the corresponding MAC addresses before packets are routed. IPv6 address resolution operation based on requires broadcast on a single-link [13]. Hence, the LSBC is again used to perform the address resolution. In a usage scenario where the FN's and MN's are highly mobile, frequent update of neighbor caches and route tables is necessary. LSBC is expected to reduce the overhead of flooding significantly.

When an IP packet originated from an Internet host and destined to an FN/MN is received by the AP that announces the route for the FN/MS, the IP layer of the AP hands over the packet to the ad hoc routing layer for multi-hop forwarding since all FN/MN's appear as if they were a single hop away from the AP. Before the route table is searched, address resolution is performed. If the destination node exists within the subnet, the MAC address is returned within address resolution reply message by unicast. Using this MAC address, the AP searches the route table for the entry of the destination node. A new route table entry is added when an outbound data packet is received by the AP and when a router solicitation message arrives at the AP. Hence, the AP does not flood RREQ messages for destination nodes within its subnet. If an entry is a stale one, the AP would receive a RRER message. In this case, the AP should flood a RREQ message to find an alternate path. Note that there is no tunneling or examination of routing headers involved in AP's forwarding a packet to a destination node in SOHAN.

In order to deliver a packet to an Internet host, the FN/MN's use the default route to forward the packet through the ad hoc network to the AP. The FN/MN's determine its destination is located outside the subnet by examining the destinations prefix. Packet forwarding between two nodes that are associated with different AP's must go through the wired links. Once the FN determines to use the default route to the AP, normal ad hoc routing proceeds in the ad hoc routing layer, which is transparent to the upper layer. The AP then forwards the packet towards the destination through the Internet as usual.

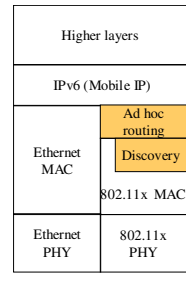

AR

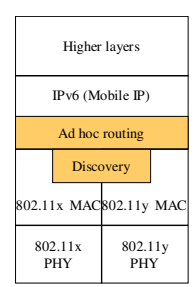

FN

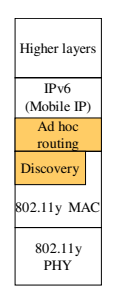

MN

Fig. 3. Protocol stack for interworking of SOHAN and Internet

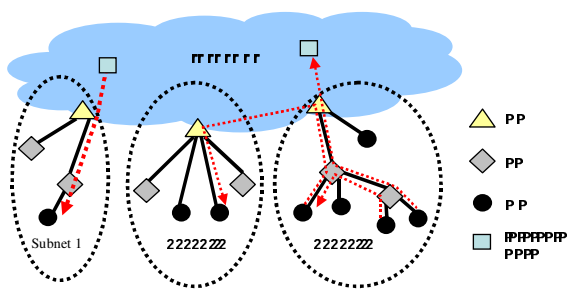

Fig. 4. Hierarchical structure of SOHAN

\section{Simulation}

\subsection{Simulation Environments}

We used ns-2 simulator with Monarch extensions to evaluate the performance of the proposed methods for interworking between SOHAN and the Internet. For the three 
tiers of SOHAN nodes, we implemented the topology discovery and routing protocols based on 802.11 MAC and AODV with appropriate modifications to operate directly on top of 802.11 MAC layer with MAC addresses. Procedures for LSBC and reverse path setup were implemented and added to the AODV module. Both IPv6 and mobile IPv6 modules were also added with the implementation of address autoconfiguration, routing, and gateway discovery. The main simulation parameters are summarized in Table 1. Five different combinations of AP's, FN's and MN's were used to study the performance with respect to the total number nodes. The number of AP's, FN's and MN's were chosen in accordance with the result by Liu et al. which states that the throughput capacity increases linearly with the number of AP's if it grows faster than the square root of the number of nodes [14]. All AP's are configured as routers and connected to a backbone router in a star topology. This backbone router is directly connected to another router to which all the Internet hosts used in the simulation are connected via a LAN. All of the wired links between the AP's and Internet hosts are given enough bandwidth (100 Mbps) that congestion does not occur in the wired section of end-to-end path between the MN and Internet host. Every communication session is established between a MN and an Internet host. That is, no traffic flows exist between two MN's or two Internet hosts.

In order to compare the performance of SOHAN in interworking with the Internet against that of flat ad hoc networks, we ran a series of simulations with the same parameters using the interworking procedures proposed by Wakikawa et al. for flat ad hoc network [8]. The flat ad hoc network consists of the AP's and MN's only. This implies all MN's are capable of forwarding packets among themselves. In each case of the simulation, the number of MN's is made equal to the number of FN's plus the number of MN's.

Table 1. Simulation Parameters

\begin{tabular}{|c|c|c|c|c|c|}
\hline Simulation area & \multicolumn{5}{|c|}{$1000 \mathrm{~m} \times 1000 \mathrm{~m}$} \\
\hline Number of AP's & 4 & 6 & 8 & 10 & 12 \\
\hline Number of FN's & 6 & 12 & 22 & 34 & 48 \\
\hline Number of MN's & 10 & 24 & 42 & 66 & 96 \\
\hline Number of Internet hosts & \multicolumn{5}{|c|}{4 pkts/sec } \\
\hline Number of pkts/sec generated & \multicolumn{5}{|c|}{20} \\
\hline Packet size & \multicolumn{5}{|c|}{ Random waypoint model $20 \mathrm{~m} / \mathrm{s}$} \\
\hline Number of communication pairs & \multicolumn{5}{|c|}{$1000 \mathrm{sec}$} \\
\hline Mobility model & \multicolumn{5}{|c|}{} \\
\hline Max speed & \multicolumn{5}{|c|}{} \\
\hline Pause time & \multicolumn{5}{|c|}{} \\
\hline Simulation time & \multicolumn{5}{|c|}{} \\
\hline
\end{tabular}

\subsection{Simulation Results}

We measure the system throughput, average end-to-end delay, and normalized overhead while increasing the number of nodes in order to compare the performance of the two networks with respect to scalability. The number of bytes is measured for MAC frames, instead of IP packets, since routing for SOHAN takes place in L2.5. 
Fig. 5(a) shows the curves for normalized control overheads. One can observe that the control overheads incurred by the flat ad hoc network increase at an exponential rate whereas they increase at a near linear rate. This indicates that flooding of control messages for gateway and route discovery in the flat ad hoc network can reduce the performance significantly. On the other hand, the amount of flood packets in SOHAN is better controlled thanks to LSBC.

Fig. 5(b) clearly indicates that SOHAN has better scalability properties than the flat network in terms of throughput. Notice that SOHAN produces lower throughput than the flat network when the number of nodes is 16. This is because SOHAN has only $10 \mathrm{AP} / \mathrm{FN}$ 's and some MN's lose connections. Since all nodes are capable of forwarding in flat networks, the loss of connection is less likely to happen. Finally, Fig. 5(c) also clearly illustrates the improved scalability property achieved by SOHAN compared to that of the flat network.

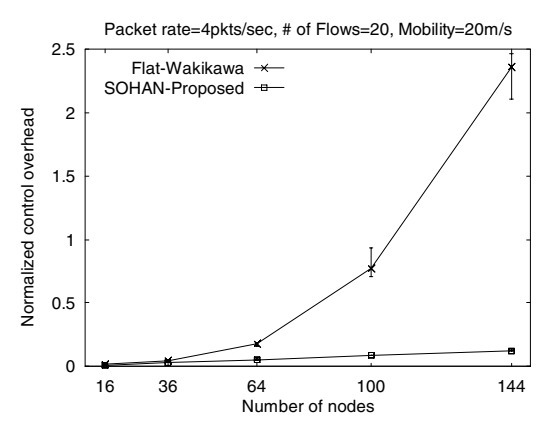

(a) Routing overhead vs. Number of nodes

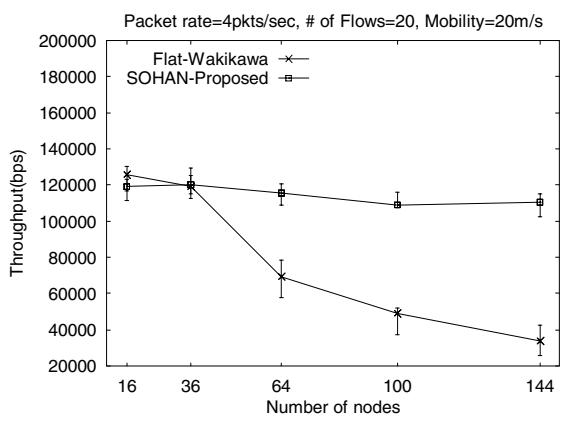

(b) Throughput vs. Number of nodes

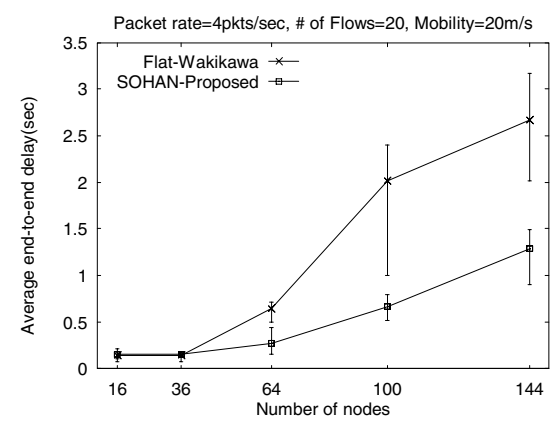

(c) Average end-to-end delay vs. Number of nodes

Fig. 5. Simulation Results

\section{Conclusions}

We presented a method of interworking with the Internet for self-organizing ad hoc networks based on IPv6. The most distinctive feature of our method is that, at the network layer ad hoc nodes perform normal IPv6 operations while interworking gateways act as a normal IPv6 access router. That is, all interworking functionalities 
along with ad hoc routing and topology discovery are hidden in the sub-IP (extended MAC) layer. This allows the interworking to be scalable as well as to exploit the hierarchical structure of SOHAN. As a result, a significant improvement in performance can be achieved in terms of control overhead, throughput and delay compared to an interworking procedure proposed for a flat ad hoc network.

\section{References}

1. Gupta, P., Kumar, P.: The Capacity of Wireless Networks. IEEE Transactions on Information Theory, Mar 2000, Vol. IT-46(2), 388-404

2. Ganu, S., Raju, L., Anepu, B., Seskar, I., Raychaudhuri, D.: Architecture and Prototyping of an 802.11-based Self-Organizing Hierarchical Ad-Hoc Wireless Network (SOHAN). submitted to MobiHoc (2004)

3. Lei, H., Perkins, C.: Ad Hoc Networking with Mobile IP. Proc. of the 2nd European Personal Mobile Communications Conference. (Oct, 1997) 197-202

4. Broch, J., Maltz, D., Johnson, D.: Supporting Hierarchy and Heterogeneous Interfaces in Multi-hop Wireless Ad hoc Networks. Workshop on Mobile Computing. (1999) 370-375

5. Jonsson, U., Alriksson, F., Larsson, T., Johansson, P., Maguire Jr. G.: MIPMANET Mobile IP for Mobile Ad Hoc Networks. MobiHOC'00. (Aug, 2000) 75-85

6. Sun, Y., Belding-Royer, E., Perkins, C.: Internet connectivity for ad hoc mobile networks. International Journal of Wireless Information Networks special issue on Mobile Ad hoc Networks. (2002) Vol. 9(2), 75-88

7. Xi, J., Bettstetter, C.: Wireless Multihop Internet Access : Gateway Discovery, Routing and Addressing. Proc. of the Int. Conf. on 3G and Beyond 3G Wireless. (May, 2002) 109114

8. Wakikawa, R., Malinen, J., Perkins, C., Nilsson, A., Tuominen, A.: Global Connectivity for IPv6 Mobile Ad Hoc Networks. draft-wakikawa-manet-globalv6-04.txt. IETF Internet Draft. (Jul, 2005)(work in progress)

9. Johnson, D., Perkins, C., Arkko, J.: Mobility Support in IPv6. IETF RFC 3775 (Jun, 2004)

10. Yang, S.: A Joint MAC Discovery-Routing Protocol for Self-Organizing Hierarchical Ad Hoc Networks. Ph.D. Thesis (2004)

11. Thomson, S., Narten, T.: IPv6 Stateless Address Autoconfiguration. IETF RFC 2462. (Dec, 1998)

12. Jeong, J., Park, J., Kim, H., Kim, D.: Ad Hoc IP Address Autoconfiguration. draft-jeongadhoc-ip-addr-autoconf-02.txt. IETF Internet Draft. (Feb, 2004)(work in progress)

13. Narten, T., Nordmark, E., Simpson, W.: Neighbor Discovery for IPv6. IETF RFC 2461. (Dec, 1998)

14. Liu, B., Liu, Z., Towsley, D.: On the Capacity of Hybrid Wireless Networks. IEEE INFOCOM '03. (Apr, 2003) Vol. 2, 1543-1552 